\title{
Utilização da Classificação Internacional de Funcionalidade, Incapacidade e Saúde em Mulheres com Câncer de Mama: Revisão da Literatura
}

https://doi.org/10.32635/2176-9745.RBC.2019v65n1.349

\author{
Use of the International Classification of Functioning, Disability, and Health in Women with Breast Cancer: Literature Review \\ Utilización de la Clasificación Internacional de Funcionalidad, Incapacidad y Salud en Mujeres con Cáncer de Mama: \\ Revisión de la Literatura
}

Valkiria de Araujo dos Santos'; Thamiris Seippel ${ }^{2}$, Luciana Castaneda ${ }^{3}$

Resumo

Introduçáo: $\mathrm{O}$ tratamento para o câncer de mama pode apresentar consequências negativas para a funcionalidade das mulheres. Projetos terapêuticos conduzidos à funcionalidade podem auxiliar na tomada de decisáo orientada às necessidades reais de mulheres com câncer de mama. Objetivo: Traçar o panorama de utilização da Classificação Internacional de Funcionalidade, Incapacidade e Saúde (CIF) como medida de desfecho da funcionalidade em mulheres com câncer de mama. Método: Revisão de literatura. A busca foi realizada nas bases de dados SciELO, LILACS e MEDLINE. A elaboração do protocolo de pesquisa visou a atender às orientaçóes das recomendações Prisma. Resultados: Foram recuperados nove artigos. Houve um predomínio de utilização de desenhos de estudo quantitativos com aplicação ou validação de listas resumidas da CIF. A metodologia de ligação de instrumentos esteve presente em quatro estudos. Um estudo utilizou apenas o modelo conceitual da CIF. No âmbito da alta incidência do câncer de mama e da cronicidade dos efeitos da própria condição de saúde e do tratamento, o número de estudos encontrados foi pequeno. Conclusáo: Foram recuperados nove estudos sobre a CIF no contexto do câncer de mama. Em todos os estudos, os autores afirmaram que a CIF é uma ferramenta fundamental para o planejamento do cuidado oncológico.

Palavras-chave: Neoplasias da Mama; Classificação Internacional de Funcionalidade, Incapacidade e Saúde; Revisão.

\begin{abstract}
Introduction: Treatment for breast cancer may have negative consequences in women functioning. Functional-oriented therapeutic designs can aid in decision-making that addresses the real needs of women with breast cancer. Objective: To outline the use of the International Classification of Functioning, Disability, and Health (ICF) as an outcome measure of functional in women with breast cancer. Method: A literature review. The search was performed in SciELO, LILACS and MEDLINE databases. The research protocol aimed at meeting Prisma Recommendations. Results: Nine articles were retrieved. There was a predominance of quantitative study designs with application or validation of ICF core sets. Linking rules were present in four studies and one study used only the ICF conceptual model. The number of studies found was small, in the context of the high incidence of breast cancer and the chronicity of the effects of the health condition itself and the treatment. Conclusion: The recovered studies were categorical when affirming that the use of the ICF is essential for the cancer care services offered to women with breast cancer.

Key words: Breast Neoplasms; International Classification of Functioning, Disability and Health; Review.
\end{abstract}

\begin{abstract}
Resumen
Introducción: El tratamiento para el cáncer de mama puede presentar consecuencias negativas para la funcionalidad de las mujeres. Los proyectos terapéuticos orientados a la funcionalidad pueden ayudar a tomar decisiones orientadas a las necesidades reales de las mujeres con cáncer de mama. Objetivo: Trazar el panorama de utilización de la Clasificación Internacional del Funcionamiento, de la Discapacidad y de la Salud (CIF) como medida de desenlace de la funcionalidad en mujeres con cáncer de mama. Método: Revisión de literatura. La búsqueda se realizó en las bases de datos SciELO, LILACS y MEDLINE. La elaboración del protocolo de investigación pretendió atender las orientaciones de las recomendaciones Prisma. Resultados: Se recuperaron nueve artículos. Hubo un predominio de utilización de dibujos de estudio cuantitativos con aplicación o validación de listas resumidas de la CIF. La metodología de enlace de instrumentos estuvo presente en cuatro estudios. Un estudio utilizó sólo el modelo conceptual de la Clasificación Internacional de Funcionalidad. En el ámbito de la alta incidencia del cáncer de mama y de la cronicidad de los efectos de la propia condición de salud y del tratamiento, el número de estudios encontrados fue pequeño. Conclusión: Los estudios recuperados fueron categóricos al afirmar que el uso del modelo biopsicosocial de la CIF es fundamental para la planificación del cuidado oncológico ofrecido a mujeres con cáncer de mama.

Palabras clave: Neoplasias de la Mama; Clasificación Internacional del Funcionamiento, de la Discapacidad y de la Salud; Revisión.
\end{abstract}

\footnotetext{
${ }^{1}$ Instituto Federal de Educação, Ciência e Tecnologia do Rio de Janeiro (IFRJ). Rio de Janeiro (RJ), Brasil. Orcid iD: https://orcid.org/0000-0001-6290-3617 ${ }^{2}$ IFRJ. Rio de Janeiro (RJ), Brasil. Orcid iD: https://orcid.org/0000-0003-3722-8589

${ }^{3}$ IFRJ. Rede Brasileira de Pesquisa em Funcionalidade. Rio de Janeiro (RJ), Brasil. Orcid iD: https://orcid.org/0000-0001-8573-342X

Endereço para correspondência: Luciana Castaneda. Rua Professor Carlos Wenceslau, 343 - Realengo. Rio de Janeiro (RJ), Brasil. CEP 21715-000.

E-mail: luciana.ribeiro@ifrj.edu.br
} 


\section{INTRODUÇÃO}

A análise de indicadores de mortalidade, incidência e sobrevida são fundamentais para a redução das iniquidades no acesso ao tratamento oncológico ${ }^{1}$. Estimativas apontam que cerca de 1,7 milhão de mulheres foram diagnosticadas com câncer de mama no ano de 2008, com 522 mil mortes relacionadas ${ }^{2}$. As evidências relatam que quase metade dos casos ocorre em regióes de baixa e média rendas. No Brasil, a característica da oferta de serviços de oncologia náo tem garantido acesso universal a programas de prevenção, sejam de prevenção primária, secundária e terciária ${ }^{3}$. Sendo assim, deixam um enorme contigente populacional de brasileiros que dependem exclusivamente do Sistema Único de Saúde (SUS) para a utilização de serviços oncológicos, descobertos pelos serviços de saúde ${ }^{4}$.

O câncer de mama é o segundo tipo de câncer mais comum e o mais frequente na população feminina, sendo a primeira causa de morte por câncer entre as mulheres. Para cada ano do biênio 2018-2019, foram estimados aproximadamente 60 mil casos, representando 29,5\% de todos os casos de câncer em mulheres no país. Sendo assim, o câncer de mama apresenta um risco de 56,33 casos a cada 100 mil mulheres 5 .

Houve um grande avanço no conhecimento sobre os indicadores de mortalidade e incidência do câncer de mama em mulheres, porém, o mesmo não se verifica quando o tema é a funcionalidade, apesar de o câncer de mama apresentar consequências negativas nessa questáo. É consenso na literatura que as repercussóes físicas, emocionais, sociais e psicológicas podem ser enfrentadas no decorrer do tratamento e após a sua conclusão ${ }^{6}$. Dor, aderência pericicatricial, infecção, necrose de pele, seroma, diminuição de amplitude de movimento do ombro, síndrome da rede axilar, lesão de nervos motor (escápula alada) e/ou sensitivo (parestesia de intercostobraquial), fraqueza muscular, alteração sensorial, linfedema e neuropatia periférica induzida por quimioterapia são algumas das possíveis manifestaçóes no ambito das funçóes e estruturas do corpo.

Associado ao tratamento com radioterapia, é possível que ocorram alteraçôes na sensibilidade, fadiga e alteraçôes do aparelho respiratório. A hormonioterapia pode levar à retenção de líquidos, ondas de calor, artralgia, prurido, secura vaginal e trombose venosa profunda $a^{7-15}$. A grande variedade de repercussóes relacionadas ao tratamento pode ter impacto nos demais componentes da funcionalidade, afetando domínios de atividades e participaçăo ${ }^{16}$. Ademais, as implicaçóes negativas de barreiras no que tange ao acesso ao tratamento precoce também são possivelmente determinantes para a redução da funcionalidade em mulheres com câncer de mama.
Há uma tendência da agenda global de incorporação de desfechos que superem os indicadores tradicionais de sobrevida e mortalidade. $\mathrm{O}$ desfecho de funcionalidade é considerado atualmente como o terceiro indicador de saúde ${ }^{17}$. Orientado aos domínios do modelo biopsicossocial (condição de saúde, funçóes do corpo, estruturas do corpo, atividades, participação, fatores ambientais e fatores pessoais), o indicador da funcionalidade pode colaborar na construçáo de projetos terapêuticos orientados às necessidades reais dos usuários ${ }^{18}$. A Classificação Internacional de Funcionalidade, Incapacidade e Saúde (CIF) é uma ferramenta de referência para a orientação do modelo biopsicossocial ${ }^{19,20}$. Sua estrutura permite: 1) avançar na utilização de linguagem em saúde homogênea; 2) ajudar na construçáo de projetos terapêuticos individualizados e orientados às necessidades reais dos usuários; 3) estruturar e fortalecer a inserção de indicadores da funcionalidade em Sistemas de Informação em Saúde ${ }^{21-23}$. Considerando as potencialidades da CIF para a orientação do cuidado oncológico, o presente estudo tem como objetivo traçar o panorama de utilizaçáo da classificaçáo como medida de desfecho da funcionalidade para mulheres com câncer de mama.

\section{MÉTODO}

Trata-se de uma revisão de literatura. A elaboração do protocolo de pesquisa visou a atender às orientaçóes das Recomendaçôes Prisma ${ }^{24}$. A estratégia de busca foi elaborada por meio do emprego de termos padronizados indexados e outros termos livres pertinentes ao tema, interligados por conectores. Foram consultadas as bases de dados LILACS, SciELO e MEDLINE. Não houve restrição quanto à data de publicação. Foram incluídos estudos em inglês, português e espanhol. Além da busca em base de dados, também foram realizadas buscas manuais e revisão das listas de referências dos estudos selecionados com objetivo de identificar outras publicaçôes. A Estratégia $\mathrm{PICO}^{24}$ foi utilizada para População: mulheres com câncer de mama; Intervençáo/exposição: terapias como cirurgia, radioterapia, terapia endócrina, quimioterapia neoadjuvante, adjuvante e paliativa, cuidados paliativos, reabilitação etc.; Comparador: qualquer alternativa às opções de terapia; e Desfecho: medidas de funcionalidade que tenham utilizado a CIF de forma direta ou indireta.

A estratégia utilizada para a base MEDLINE foi: (((Breast Neoplasms) OR ((breast[Title/Abstract] OR mammary*[Title/ Abstract]) AND (neoplasm*[Title/Abstract] OR cancer*[Title/ Abstract] OR carcinoma*[Title/Abstract] OR tumor*[Title/ Abstract] OR tumor*[Title/Abstract] OR onco*[title/ abstrac]))) AND functioning[Title]) AND international classification of functioning[Title/Abstract]))). 
Os critérios de inclusão foram: estudos sobre o tema de mulheres com câncer de mama confirmado; estudos originais; estudos que tenham utilizado a CIF (de forma direta ou indireta). Os critérios de exclusão foram: estudos com câncer de mama não primário; estudos que tinham apenas o resumo; outros tipos de publicaçóes: palestras, opiniáo etc. As referências foram exportadas para um gerenciador de referência (Mendeley).

Dois revisores independentes (VA \& TS) foram responsáveis por avaliar e selecionar inicialmente os títulos e os resumos. Os desacordos no processo de revisão dos resumos e textos completos de artigos que corresponderam aos critérios de elegibilidade foram analisados e resolvidos por um terceiro revisor (LC). As seguintes informaçóes das publicações foram extraídas: detalhes da publicação (autor, instituição, ano de publicação), questão de pesquisa (cenário básico), população, intervenção/ comparador, metodologia da CIF utilizada (checklist, core set, WHODAS etc).

\section{RESULTADOS}

Onze estudos foram recuperados nas buscas das bases de dados; nove atenderam aos critérios de inclusão (Figura 1). Os estudos foram majoritariamente desenhos observacionais do tipo transversal. Também foram encontrados estudos Delphi e identificados dois estudos do tipo revisão, sendo uma revisão sistemática e uma revisão da literatura. Foi incluído um estudo qualitativo do tipo grupo focal (Quadro 1).

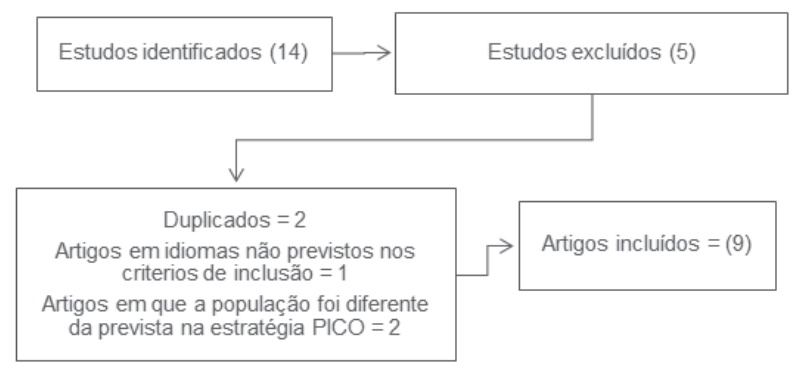

Figura 1. Fluxograma da revisão

A caracterização da amostra foi, em sua maioria, de mulheres com câncer de mama. Também foi possível observar amostras compostas por especialistas no tema. O estudo de revisão sistemática incluiu apenas ensaios clínicos randomizados como desenho válido para inclusão no protocolo. Observou-se um predomínio de cinco estudos que utilizaram desenhos de estudo quantitativos com aplicação ou validação dos core sets. As metodologias de ligaçáo de instrumentos, percepçóes ou relatos de usuários com as categorias da CIF estiveram presentes em quatro dos nove estudos (44\%). Um estudo utilizou apenas o modelo conceitual da CIF (11\%).

\section{DISCUSSÃO}

O presente estudo teve como objetivo traçar a perspectiva de utilização da CIF no câncer de mama como medida de desfecho da funcionalidade. As perspectivas adotadas no estudo evidenciam que diversas formas de utilização da linguagem do modelo e do sistema de classificação da CIF são possíveis. No âmbito de publicaçôes brasileiras sobre o tema, Carvalho et al. ${ }^{28}$ identificaram e discutiram instrumentos específicos para câncer de mama já validados para o Brasil e traçaram uma analogia com o core set abrangente para câncer de mama. Os mesmos autores ainda realizaram um estudo observacional transversal em mulheres com câncer de mama em um hospital de referência do Rio de Janeiro. O estudo utilizou apenas o modelo conceitual da CIF com a aplicação de um instrumento genérico de avaliaçáo da incapacidade do membro superior Shoulder and Hand Questionnaire (DASH), um questionário de suporte social e o World Health Organization Quality of Life (WHOQOL) para descrever o fenômeno na funcionalidade ${ }^{29}$. Cabe aqui a discussão sobre a diferenciaçáo do conceito de Qualidade de Vida Relacionada à Saúde (QVRS) abordado pelo WHOQOL e o conceito de funcionalidade abordado pela CIF.

Embora se referiram a experiências humanas, trata-se de fenômenos distintos. A QVRS tem sido amplamente consolidada na literatura de cuidado a saúde como uma importante medida de desfecho no âmbito do cuidado oncológico. Visa à experiência subjetiva dos indivíduos em relaçáo ao bem-estar, grau com que as pessoas aproveitam as possibilidades de suas vidas e a percepçáo de satisfação com a vida. Já a funcionalidade é definida como uma experiência humana relacionada às condiçôes de saúde, funçôes e estruturas do corpo, atividades e participação e a interação desses domínios com fatores contextuais (fatores pessoais e ambientais) ${ }^{20}$. As metodologias de ligação de instrumentos, conteúdos de falas de usuárias e de percepçáo dos especialistas com as categorias da CIF se destacaram nos estudos incluídos. Denominada Linking Rules $^{33}$, tem sido um método amplamente utilizado desde a publicação da primeira proposta de ligação de informações em saúde com o sistema alfanumérico da CIF $^{34}$. A metodologia oferece a possibilidade de conhecer quais domínios do modelo biopsicossocial estão presentes nos instrumentos de avaliação de desfecho. Além disso, traz para os profissionais de saúde, gestores e pesquisadores a capacidade de efetivamente elucidar quais 
Quadro 1. Características dos estudos incluídos na revisão

\begin{tabular}{|c|c|c|c|c|}
\hline Autor & Objetivos & $\begin{array}{l}\text { Desenho de } \\
\text { estudo }\end{array}$ & $\begin{array}{c}\text { Caracterização da } \\
\text { amostra }\end{array}$ & $\begin{array}{l}\text { Forma de } \\
\text { utilização da } \\
\text { CIF }\end{array}$ \\
\hline Khan et al. ${ }^{15}$ & $\begin{array}{l}\text { Examinar a relevância e aplicabilidade } \\
\text { do core set da CIF para o câncer de } \\
\text { mama }\end{array}$ & Transversal & $\begin{array}{l}85 \text { mulheres com } \\
\text { câncer de mama }\end{array}$ & $\begin{array}{c}\text { Core set } \\
\text { abrangente }\end{array}$ \\
\hline Glaessel et al. ${ }^{25}$ & $\begin{array}{l}\text { Examinar a validade do conteúdo do } \\
\text { core set da CIF para o câncer de mama } \\
\text { sob a perspectiva dos fisioterapeutas }\end{array}$ & $\begin{array}{l}\text { Consenso de } \\
\text { especialistas }\end{array}$ & $\begin{array}{l}59 \text { fisioterapeutas } \\
\text { especialistas }\end{array}$ & $\begin{array}{c}\text { Core set } \\
\text { abrangente }\end{array}$ \\
\hline Brockow et al. ${ }^{26}$ & $\begin{array}{c}\text { Identificar e quantificar os conceitos } \\
\text { contidos nas medidas de resultado de } \\
\text { ensaios clínicos randomizados para } \\
\text { intervenções no câncer de mama, } \\
\text { usando a CIF como uma ferramenta de } \\
\text { referência }\end{array}$ & $\begin{array}{l}\text { Revisão } \\
\text { sistemática }\end{array}$ & $\begin{array}{l}640 \text { ensaios clínicos } \\
\text { randomizados }\end{array}$ & $\begin{array}{l}\text { Método de } \\
\text { ligação }\end{array}$ \\
\hline $\begin{array}{l}\text { Carvalho et } \\
\text { al. }^{27}\end{array}$ & $\begin{array}{l}\text { Identificar e discutir os instrumentos } \\
\text { validados para o português capazes de } \\
\text { mensurar códigos do core set da CIF } \\
\text { para câncer de mama }\end{array}$ & $\begin{array}{l}\text { Revisão da } \\
\text { literatura }\end{array}$ & 15 instrumentos & $\begin{array}{l}\text { Método de } \\
\text { ligação }\end{array}$ \\
\hline $\begin{array}{l}\text { Carvalho et } \\
\text { al. }^{28}\end{array}$ & $\begin{array}{l}\text { Avaliar a funcionalidade de mulheres } \\
\text { diagnosticadas com câncer de mama de } \\
\text { acordo com a CIF }\end{array}$ & Transversal & $\begin{array}{l}105 \text { mulheres com } \\
\text { câncer de mama }\end{array}$ & Conceitual \\
\hline Yang et al. ${ }^{29}$ & $\begin{array}{c}\text { Desenvolver um questionário } \\
\text { autopreenchido com os itens do } \\
\text { questionário curto do core set para } \\
\text { o câncer de mama (BCSQ-BC) e } \\
\text { investigar a prevalência de disfunções } \\
\text { específicas ao longo do curso do câncer } \\
\text { e tratamentos }\end{array}$ & Transversal & $\begin{array}{l}96 \text { mulheres com } \\
\text { câncer de mama }\end{array}$ & $\begin{array}{l}\text { Core set } \\
\text { resumido }\end{array}$ \\
\hline Cooney et al. ${ }^{30}$ & $\begin{array}{c}\text { Validar o core set da CIF para o câncer } \\
\text { de mama a partir da perspectiva de } \\
\text { mulheres com câncer }\end{array}$ & Grupo Focal & $\begin{array}{l}34 \text { mulheres com } \\
\text { câncer de mama }\end{array}$ & $\begin{array}{l}\text { Método de } \\
\text { ligação }\end{array}$ \\
\hline Yang et al. ${ }^{31}$ & $\begin{array}{c}\text { Construir um instrumento clínico } \\
\text { para avaliar a funcionalidade em } \\
\text { sobreviventes de câncer de mama, } \\
\text { usando CIF para funções do corpo, } \\
\text { atividade e participação e fatores } \\
\text { ambientais }\end{array}$ & Transversal & $\begin{array}{l}158 \text { mulheres com } \\
\text { câncer de mama }\end{array}$ & $\begin{array}{l}\text { Core set } \\
\text { resumido }\end{array}$ \\
\hline Letellier et al. ${ }^{32}$ & $\begin{array}{l}\text { Estimar a extensão em que o conteúdo } \\
\text { dos EORTC QLQ-C30 e EORTC QLQ- } \\
\text { BR23 vai além do funcionamento e } \\
\text { inclui sensação global de bem-estar }\end{array}$ & $\begin{array}{l}\text { Consenso de } \\
\text { especialistas }\end{array}$ & 34 especialistas & $\begin{array}{l}\text { Método de } \\
\text { ligação }\end{array}$ \\
\hline
\end{tabular}

Legenda: CIF = Classificação Internacional de Funcionalidade, Incapacidade e Saúde.

domínios específicos da funcionalidade estão presentes nos protocolos de avaliação. E, dessa forma, propor a complementação com outras medidas de desfecho do modelo biopsicossocial ${ }^{35}$.

Quatro dos nove estudos testaram ou validaram os core sets (abrangente e reduzido) para câncer de mama. Desde 2004, a Organização Mundial da Saúde emprega esforços na construção e disseminação dessas ferramentas para facilitar a utilização clínica da CIF $^{36}$. Em um estudo de revisão sistemática sobre os core sets e sua aplicação, a área da oncologia não figura entre as que tiveram maior número de publicações sobre o tema, destacando-se a área da Neurologia ${ }^{37}$. Para facilitar o uso prático, ferramentas baseadas na CIF devem ser adaptadas para as necessidades de potenciais usuários, sem desqualificar os padrôes para a coleta de informação necessária para as estatísticas de saúde e sistemas de informaçãa ${ }^{38}$.

A estrutura, oferta e acessos aos serviços oncológicos variam entre os países em função de seus sistemas de saúde e legislações de Seguridade Social, embora haja um amplo consenso em relação a seus principais objetivos ${ }^{39}$. O objetivo de resgatar/otimizar a funcionalidade, sem dúvida, figura entre os principais. Refletir sobre as razóes da baixa utilizaçáo da CIF na reabilitação em oncologia, embora 
se faça eminentemente necessário, não foi o escopo deste trabalho, apesar de ter sido um achado bastante relevante e serem encontrados apenas nove estudos que utilizaram o modelo da CIF nas publicações sobre mulheres com câncer de mama.

A limitação do presente trabalho se dá pela inclusão de apenas três bases de dados para a busca, o que caracteriza o estudo como não sendo de revisão sistemática. No entanto, as demais recomendaçóes Prisma que visavam à dupla avaliação por pares tanto dos resumos quanto da extraçáo dos resultados foi seguida, aumentando potencialmente a validade interna dos achados. As conclusôes dos estudos incluídos na revisão chegam ao senso comum de que o papel da CIF como medida de desfecho da funcionalidade é imprescindível para avaliação, tratamento e efetividade de condutas adotadas no cuidado ofertado a mulheres com câncer de mama.

A baixa utilização da CIF na oncologia pode estar relacionada com o desconhecimento, impressão de alta complexidade da ferramenta, desinteresse profissional, baixo envolvimento da gestáo, além de baixa iniciativa macroinstitucional. Outro fator que pode colaborar é a dificuldade de aplicação da CIF com o sistema de $\operatorname{códigos}^{40}$. A utilizaçấo do sistema de codificação da CIF está intimamente relacionada à construção e ao fortalecimento de sistemas de informação em saúde ${ }^{41}$ e à utilização de forma complementar à Classificação Internacional de Doenças (CID) ${ }^{42}$. Na versão da CID-11 prevista para 2019, um capítulo de informaçóes da funcionalidade será incorporado ${ }^{43}$. Parece não haver possibilidades futuras para a não utilização da classificação. Estratégias de incorporação do modelo de orientação da funcionalidade e da linguagem da CIF, sem a necessidade de incorporaçáo imediata dos códigos, podem ser um caminho para a consolidação da mudança de paradigma do modelo biomédico para o modelo biopsicossocial.

\section{CONCLUSÃO}

A atividade científica sobre a CIF no câncer de mama foi mapeada no presente estudo, tendo recuperado um pequeno número de evidências científicas. Nove estudos abordaram a temática de utilização da CIF como medida de desfecho da funcionalidade no cenário do cuidado oncológico para mulheres com câncer de mama. Os estudos variaram quanto ao desenho metodológico, forma de aplicação da CIF, além das características da amostra. No entanto, todos os estudos foram categóricos ao afirmar em suas conclusóes que o modelo biopsicossocial é fundamental para o planejamento do cuidado oncológico ofertado a mulheres com câncer de mama.

\section{CONTRIBUIÇÕES}

Luciana Castaneda participou da concepção e planejamento do estudo, análise e interpretação dos dados e redaçáo do manuscrito. Valkiria de Araujo dos Santos participou da análise e interpretação dos dados e redação do manuscrito. Thamiris Seippel participou da análise e interpretação dos dados. Todas as autoras aprovaram a versão final do artigo.

\section{DECLARAÇÃO DE CONFLITO DE INTERESSES}

Nada a declarar.

\section{FONTES DE FINANCIAMENTO}

Não há.

\section{REFERÊNCIAS}

1. Pilleron S, Sarfati D, Janssen-Heijnen M, et al. Global cancer incidence in older adults, 2012 and 2035: a population-based study. Int J Cancer. 2019;144(1):4958. doi: https://doi.org/10.1002/ijc.31664.

2. Tao Z, Shi A, Lu C, et al. Breast cancer: epidemiology and etiology. Cell Biochem Biophys. 2015 Jun;72(2):333-8. doi: https://doi.org/10.1007/s12013-014-0459-6.

3. Silva GA, Souza-Júnior PRB, Damacena GN, et al. Early detection of breast cancer in Brazil: data from the National Health Survey, 2013. Rev Saúde Pública. 2017;51(Suppl 1):14s. doi: http://dx.doi.org/10.1590/ s1518-8787.2017051000191.

4. Bukowski A, Gioia S, Chavarri-Guerra Y, et al Patient Navigation to Improve Access to Breast Cancer Care in Brazil. J Glob Oncol. 2017;3(5):433-437. doi: http:// dx.doi.org/10.1200/JGO.2016.006726.

5. Instituto Nacional de Câncer José Alencar Gomes da Silva. Estimativa 2018: incidência de câncer no Brasil [Internet]. Rio de Janeiro: INCA, 2017. [acesso 2018 nov 15]. Disponível em: http://www.inca.gov.br/estimativa/2018.

6. Lee KM, Jung D, Hwang $\mathrm{H}$, et al. Pre-treatment anxiety is associated with persistent chemotherapyinduced peripheral neuropathy in women treated with neoadjuvant chemotherapy for breast cancer. J Psychosom Res. 2018;108: 14-19. doi: https://doi. org/10.1016/j.jpsychores.2018.02.012

7. Leysen L, Adriaenssens N, Nijs J, et al. Chronic pain in breast cancer survivors: nociceptive, neuropathic, or central sensitization pain? Pain Pract. 2018 Sep 30. Epub ahead of print. PubMed PMID: 3026943. doi: https:// doi.org/10.1111/papr.12732.

8. Maiorano E, Regan MM, Viale G, et al. Prognostic and predictive impact of central necrosis and fibrosis 
in early breast cancer: results from two International Breast Cancer Study Group randomized trials of chemoendocrine adjuvant therapy. Breast Cancer Res Treat. 2010;121(1):211-8. doi: https://doi.org/10.1007/ s10549-009-0360-y.

9. Rivera DR, Ganz PA, Weyrich MS, et al. Chemotherapyassociated peripheral neuropathy in patients with earlystage breast cancer: a systematic review. J Natl Cancer Inst. 2018;110(2):djx140. doi: https://doi.org/10.1093/ jnci/djx140.

10. Leonardis JM, Diefenbach BJ, Lyons DA, et al. The influence of reconstruction choice and inclusion of radiation therapy on functional shoulder biomechanics in women undergoing mastectomy for breast cancer. Breast Cancer Res Treat. 2018 Oct 16. Epub ahead of print. PubMed PMID: 30328049. doi: https://doi. org/10.1007/s10549-018-5003-8.

11. Thomaz JP, Dias TSM, Rezende LF. Effect of taping as treatment to reduce breast cancer lymphedema: literature review. J Vasc Bras. 2018;17(2):136-140. doi: http:// dx.doi.org/10.1590/1677-5449.007217.

12. Jang CE, Jung MS, Sohn EH, et al. The evaluation of changes in peripheral neuropathy and quality-of-life using low-frequency electrostimulation in patients treated with chemotherapy for breast cancer: a study protocol. Trials. 2018;19(1):526. doi: https://doi.org/10.1186/ s13063-018-2874-2.

13. Santos DE, Rett MT, Mendonça ACR, et al. Efeito da radioterapia na funçáo pulmonar e na fadiga de mulheres em tratamento para o câncer de mama. Fisioter Pesq. 2013:20(1):50-55. doi: http://dx.doi.org/10.1590/ S1809-29502013000100009.

14. Faiz AS, Guo S, Kaveney A, et al. Venous thrombosis and breast cancer in older women: Racial differences in risk factors and mortality. Thromb Res. 2018; 71:130-135. doi: https://doi.org/10.1016/j.thromres.2018.10.002.

15. Khan F, Amatya B, Ng L, et al. Relevance and completeness of the, International Classification of Functioning, Disability and Health (ICF) comprehensive breast cancer core set: the patient perspective in an Australian community cohort. J Rehabil Med. 2012;44(7):570-80. doi: https://doi.org/10.2340/16501977-0972.

16. Bornbaum CC, Doyle PC, Skarakis-Doyle E, et al. A critical exploration of the International Classification of Functioning, Disability, and Health (ICF) framework from the perspective of oncology: Recommendations for revision. J Multidiscip Healthc. 2013;6:75-86. doi: https://doi.org/10.2147/JMDH.S40020.

17. Stucki G, Bickenbach J. Functioning: the third health indicator in the health system and the key indicator for rehabilitation. Eur J Phys Rehabil Med. 2017;53(1):134-8. doi: https://doi.org/10.23736/S1973-9087.17.04565-8.

18. Stucki G, Reinhardt JD, Grimby G, et al. Developing "Human functioning and rehabilitation research" from the comprehensive perspective. J Rehabil Med. 2007;39(9):655-71. doi: https://doi. org/10.2340/16501977-0136.

19. Jette AM. Toward a common language for function, disability, and health. Phys Ther. 2006;86(5):726-34. doi: https://doi.org/10.1093/ptj/86.5.726.

20. Talo SA, Rytökoski UM. BPS-ICF model, a tool to measure biopsychosocial functioning and disability within icf concepts: theory and practice updated. Int J Rehabil Res. 2016;39(1):1-10. doi: https://doi. org/10.1097/MRR.0000000000000151.

21. Harty M, Griesel M, van der Merwe A. The ICF as a common language for rehabilitation goal-setting: Comparing client and professional priorities. Health Qual Life Outcomes. 2011;9:87. doi: https://doi. org/10.1186/1477-7525-9-87.

22. Castaneda L. International classification of functioning, disability and health (ICF) - way to health promotion. Rev Bras Cineantropom Desempenho Hum. 2018;20(2):229-233. doi: http://dx.doi. org/10.5007/1980-0037.2018v20n2p229.

23. Galvão TF, Pansani TS, tradutor. Principais itens para relatar revisóes sistemáticas e meta-análises: a recomendação PRISMA. Epidemiol Serv Saúde. 2015;24(2):335-342. doi: http://dx.doi.org/10.5123/ S1679-49742015000200017.

24. Santos CMC, Pimenta CAM, Nobre MRC. The PICO strategy for the research question construction and evidence search. Rev Latino-Am Enfermagem. 2007;15(3):508-11. doi: http://dx.doi.org/10.1590/ S0104-11692007000300023.

25. Glaessel A, Kirchberger I, Stucki G, et al. Does the Comprehensive International Classification of Functioning, Disability and Health (ICF) Core Set for Breast Cancer capture the problems in functioning treated by physiotherapists in women with breast cancer? Physiotherapy. 2011;97(1):33-46. doi: https://doi. org/10.1016/j.physio.2010.08.010.

26. Brockow T, Duddeck K, Geyh S, et al. Identifying the concepts contained in outcome measures of clinical trials on breast cancer using the international classification of functioning, disability and health as a reference. J Rehabil Med. 2004:44(Suppl):43-48.

27. Carvalho FN, Koifman RJ, Bergmann A. International Classification of Functioning, Disability, and Health in women with breast cancer: a proposal for measurement instruments. Cad. Saúde Pública. 2013;29(6):1093. doi: http://dx.doi.org/10.1590/ S0102-311X2013000600005.

28. Carvalho FN, Bergmann A, Koifman RJ. Functionality in women with breast cancer: the use of International Classification of Functioning, Disability and Health (ICF) in clinical practice. J Phys Ther Sci 2014;26(5):721730. doi: https://doi.org/10.1589/jpts.26.721. 
29. Yang EJ, Kim BR, Shin HI, et al. Use of the International Classification of Functioning, Disability and Health as a Functional Assessment Tool for Breast Cancer Survivors. J Breast Cancer. 2012;15(1):43-50. doi: http://dx.doi. org/10.4048/jbc.2012.15.1.43.

30. Cooney M, Galvin R, Connolly E, et al. The International Classification of Functioning (ICF) Core Set for breast cancer from the perspective of women with the condition. Disabil Rehabil. 2013;35(9):740-748. doi: https://doi. org/10.3109/09638288.2012.707742.

31. Yang EJ, Shin EK, Lim JY. Psychometric properties of scale constructed from the International Classification of Functioning, Disability and Health (ICF) core set for breast cancer based on Rasch analysis. Support Care Cancer. 2014;22(10):2839-49. doi: https://doi. org/10.1007/s00520-014-2277-1.

32. Lettelier ME, Dawes D, Mayo N. Content verification of the EORTC QLQ-C30/EORTC QLQ-BR23 with the International Classification of Functioning, Disability and Health. Qual Life Res. 2015;24(3):757-768. doi: https://doi.org/10.1007/s11136-014-0791-8.

33. Cieza A, Fayed N, Bickenbach J, et al. Refinements of the ICF Linking Rules to strengthen their potential for establishing comparability of health information. Disabil Rehabil. 2019;41(5):574-583. doi: https://doi.org/10.3 109/09638288.2016.1145258.

34. Madden RH, Bundy A. The ICF has made a difference to functioning and disability measurement and statistics. Disabil Rehabil. 2018;41(12):1450-1462. doi: https:// doi.org/10.1080/09638288.2018.1431812.

35. Üstün B, Chatterji S, Kostanjsek N. Comments from who for the journal of rehabilitation medicine special supplement on ICF core sets. J Rehabil Med. 2004;(Suppl. 44):7-8.

36. Yen TH, Liou TH, Chang KH, et al. Systematic review of ICF core set from 2001 to 2012. Disabil Rehabil. 2014;36(3):177-84. doi: https://doi.org/10.3109/0963 8288.2013.782359.

37. McIntyre A, Tempest $S$. Two steps forward, one step back? a commentary on the disease-specific core sets of the International Classification of Functioning, Disability and Health (ICF). Disabil Rehabil. 2007;29(18):1475-1479. doi: https://doi.org/10.1080/09638280601129181.

38. Castaneda L, Bergmann A, Bahia L. The International Classification of Functioning, Disability and Health: a systematic review of observational studies. Rev Bras Epidemiol. 2014;17(2):437-51. doi: http://dx.doi. org/10.1590/1809-4503201400020012ENG.

39. World Health Organization. WHO's fight against cancer: strategies that prevent, cure and care. Geneva: WHO Press; 2007.

40. Castro SS, Castaneda L, Araújo ES, et al. Aferição de funcionalidade em inquéritos de saúde no Brasil: discussão sobre instrumentos baseados na Classificação
Internacional de Funcionalidade, Incapacidade e Saúde (CIF). Rev Bras Epidemiol. 2016;19(3):65-72. doi: http://dx.doi.org/10.1590/1980-5497201600030018

41. Stucki G, Bickenbach J, Melvin J. Strengthening Rehabilitation in Health Systems Worldwide by Integrating Information on Functioning in National Health Information Systems. Am J Phys Med Rehabil. 2017;96(9):677-81. doi: http://dx.doi.org/10.1097/ PHM.0000000000000688.

42. Di Nubila HBV, Buchalla CM. O papel das Classificações da OMS - CID e CIF nas definiçôes de deficiência e incapacidade. Rev Bras Epidemiol. 2008;11(2):324-335. doi: http://dx.doi.org/10.1590/ S1415-790X2008000200014.

43. Selb M, Kohler F, Nicol MMR, et al. ICD-11: a comprehensive picture of health, an update on the ICDICF joint use initiative. J Rehabil Med. 2015;47(1):2-8. doi: http://dx.doi.org/10.2340/16501977-1928.

Recebido em 28/1/2019 Aprovado em 21/5/2019 